\title{
Security against eavesdropping in quantum cryptography
}

\author{
Norbert Lütkenhaus and Stephen M. Barnett \\ University of Strathclyde, Glasgow G4 0NG, Scotland \\ EMail: \{norbert, steve\}@phys.strath.ac.uk
}

25. September 1996

\section{Introduction to quantum cryptography}

Quantum cryptography is a method for providing two parties who want to communicate securely with a secret key to be used in established protocols of classical cryptography. For more reviews of this topic see [1, 2, 3]. Bennett and Brassard showed that it is possible, at least ideally, to create a secret key, shared by sender and receiver, without both parties sharing any secret beforehand. We refer to this protocol as the BB84 protocol. [4] To achieve this goal, sender and receiver are linked by two channels. The first channel is a public channel. The information distributed on it is available to both parties and to a potential eavesdropper. To demonstrate the principle of quantum cryptography we assume that the signals on this channel can not be changed by third parties. The second channel is a channel with strong quantum features. An eavesdropper can interact with the signal in an effort to extract information about the signals. The signal states are chosen in such a way that there is always, on average, a back reaction onto the signal states. We assume the quantum channel to be noiseless and perfect so that the back reaction of the eavesdropper's activity manifests itself as an induced error rate in the signal transmission.

The BB84 protocol uses the polarisation states of single photons as signal states. The signal states are, for example, linear vertical or horizontal polarised photons or right or left circular polarised photons. The sender sends a sequence of single photons with a polarisation chosen randomly from the four given ones. The receiver uses randomly one out of two given polarisation analysers for each signal photon. One of the analysers distinguishes between the two linear polarisations, the other between the circular polarisations. Therefore the sequence of signals contains two types of transmissions. In the first type the photon is prepared in a polarisation state which the polarisation analyser, chosen by the receiver, is able to distinguish unambiguously. An example is that a horizontal polarised photon is sent and the receiver chooses to use the linear polarisation analyser. Signals 
of this type will be refered to as deterministic signals since, the outcome of the polarisation measurement is fully determined by the state of the signal photon. The remaining signals are non-deterministic signals. An example for this is a horizontal linear polarised photon which triggers with equal probability the outcome "right circular" and "left circular" in the polarisation analyser distinguishing in the circular polarisation basis. Sender and receiver can distinguish between deterministic and non-deterministic signals using the public channel without giving away any information about the specific signal. They just compare the polarisation basis of the signal and the measuring polarisation analyser, both of which can be "linear" or "circular". The signal sequence of the deterministic bits can then be transformed into a binary key by assigning " 0 " for linear horizontal or right circular polarised photons and " 1 " for the remaining linear vertical or left circular polarised photons.

In this idealisation the security of quantum cryptography is given by the fact that the four polarisation states are not four orthogonal states and so there exists no quantum nondemolition measurement which could distinguish between them. Even more importantly, each attempt to distinguish between any of the states will change, in average, the states of the signals. This state-change destroys the perfect correlation between the signal and the measurement outcomes for the deterministic signals. A test checking this correlation will reveal any attempt at eavesdropping. If the test shows that the correlation is still perfect then sender and receiver can be sure that there was no eavesdropping attack and their shared binary key is perfectly secret.

In the practical realisation of quantum cryptography we face two specific problems. The public channel needs to be implemented in such a way that sender and receiver can ensure that the messages being received are really coming from each other. This is the problem of authentication for which various techniques are in use. In general, however, there will be the need for the two parties to share a limited amount of secret knowledge, for example in form of a secret key, before the authentication can take place. Quantum cryptography then generates a large secret key from a small secret key.

The work presented here deals with the second problem arising from the fact that all realistic quantum channels are noisy. Therefore the correlation between the signals and the measurement outcomes for the deterministic signals will not be perfect. Noise has the same effect on the signals as the activity of an eavesdropper. It is therefore necessary to think of all state change of the signals to be due to eavesdropping activity. It is intuitively clear that an eavesdropper can only have gained a small amount of information on the key if the correlation tested by sender and receiver are still strong, that is, if there are only a few transmission errors for the deterministic signals. One can hope to give a bound on the eavesdropper's Shannon information as a function of the error rate in the deterministic signals. Such bounds have been obtained assuming that the eavesdropper is restricted to von Neumann measurements only [5] or to a restricted class of more general measurements [6]. Here we present a sharp bound [7, 8] on the Shannon information of an eavesdropper which is valid for all eavesdropping attacks which access each signal photon independently 
of each other. It therefore does not include coherent attacks in which the product state of all signal photons is attacked. The sharp bound does not take into account that an eavesdropper can make use of the later acquired knowledge about the polarisation basis of the signal photons to change the measurement of the signal. However, we are able to give a rough upper bound for this situation. The reason that this is possible is that the eavesdropper has to decide how to interact with the signal states before he acquires the additional knowledge about the polarisation basis.

\section{Generalised measurements}

The key input to derive the bounds given in this paper is the most general description of a measurement on a given system. Any measurement can be described by a set of operators $A_{l}$ defined on the Hilbert space $\mathcal{H}$ of the system the measurement is performed on. The only restriction on the operators is that

$$
\sum_{l \in K} A_{l}^{\dagger} A_{l}=\mathbb{1}_{\mathcal{H}}
$$

where $K$ is some finite or countable infinite index set. The link between these operators and a measurement is given by the following formulas which describe the probabilities that a particular outcome of a measurement is triggered, and which give the final state of the measured system after that outcome was registered. For the sake of simplicity we assume the set of outcomes to be discrete. The probability that the outcome $k$ is triggered by an input state with density matrix $\rho$ is given by

$$
p_{k}=\operatorname{Tr}_{\mathcal{H}}\left(\rho \sum_{l \in K_{k}} A_{l}^{\dagger} A_{l}\right)
$$

where the $K_{k}$ are disjunct subsets of $K$ with $K=\bigcup_{k} K_{k}$. The final density matrix $\tilde{\rho}^{(k)}$ of the selected states belonging to this outcome is given by

$$
\tilde{\rho}^{(k)}=\frac{\sum_{l \in K_{k}} A_{l} \rho A_{l}^{\dagger}}{\operatorname{Tr}_{\mathcal{H}}\left(\rho \sum_{l \in K_{k}} A_{l}^{\dagger} A_{l}\right)} .
$$

The density matrix of the final state, which does not select any states, but describes the whole ensemble for all outcomes is given by

$$
\tilde{\rho}=\sum_{k} p_{k} \tilde{\rho}^{(k)}=\sum_{l \in K} A_{l} \rho A_{l}^{\dagger}
$$

It is important to choose the correct Hilbert space $\mathcal{H}$ to describe the measurement. To describe a spin measurement of an electron and the back reaction onto that spin we will 
choose $\mathcal{H}$ to be the Hilbert space of the spin of electron. If we are interested in the position or momentum of the electron as well we have to add the Hilbert space of spatial modes. It is a bit less obvious in quantum optics. If $\mathcal{H}$ is the one-photon polarisation Hilbert space then we implicitly assume that precisely one photon remains after the measurement. An eavesdropper may absorb the photon and so the final state is the vacuum. Therefore the adequate Hilbert space is the full Hilbert space of a light mode plus the polarisation degree of freedom. It turns out that we can derive the bounds presented here by restricting the Hilbert space to that of a single photon and later generalise it to the full Hilbert space.

\section{Estimate of the Shannon information}

The relevant expression for the Shannon information per signal is given with the help of the function $h(x)=-x \log x$, where $\log$ refers to basis 2 , as

$$
I=\sum_{\Psi=0,1} h[p(\Psi)]+\sum_{\substack{\alpha=o,+k}} h\left[p\left(k_{\alpha}\right)\right]-\sum_{\substack{\Psi=0,1 \\ k, \alpha}} h\left[p\left(\Psi, k_{\alpha}\right)\right] .
$$

An eavesdropper gains this Shannon information on the binary key whose signals are given by $\Psi=0,1$ when he learns, from communication on the public channel, the polarisation basis $\alpha=0,+$ (linear or circular) used for each signal, and registers the outcome $k$ on the measurement apparatus triggered by each signal. The probabilities that a " 0 " or a " 1 " is sent are denoted by $p(\Psi)$, the probability that outcome $k$ is triggered by a photon prepared as basis state of the linear or circular polarisation basis is written as $p\left(k_{\alpha}\right)$, and the joint probability distribution for both events is $p\left(\Psi, k_{\alpha}\right)$.

We would like to give an upper bound on this Shannon information as a function of the measured disturbance of the quantum channel given as the error rate in the deterministic signals. This error rate is basically the fidelity measure $D_{\text {fid }}$ of the channel, given by

$$
D_{\text {fid }}=1-\frac{1}{4} \sum_{j=1}^{4} \operatorname{Tr}_{\mathcal{H}}\left(\rho_{i} \tilde{\rho}_{i}\right) .
$$

The definition of $D_{\text {fid }}$ assumes here the use of the one photon polarisation Hilbert space. The expression $\operatorname{Tr}_{\mathcal{H}}\left(\rho_{i} \tilde{\rho}_{i}\right)$ is the overlap between the input state $\rho_{i}$, which is one of the four signal states, and the final state $\tilde{\rho}_{i}$ of the eavesdropper's measurement performed on this input state $\rho_{i}$. This can be interpreted as the probability that the final state is still recognised as the initial state in apparatus of the receiver. Then $D_{\text {fid }}$ is the error rate averaged over the four signal states.

It can be shown [8] that the optimal eavesdropping strategy can be given by operators $A_{k}$ which can be described by real matrices in a representation for which the signal states are real density matrices. Each operator $A_{k}$, which can always be expressed in the form

$$
A_{k}=\sqrt{a_{k}} O_{k}+\left(\sqrt{b_{k}}-\sqrt{a_{k}}\right) O_{k} P_{k}
$$


with real, positive numbers $a_{k}$ and $b_{k},\left(b_{k} \geq a_{k}\right)$, projection operators $P_{k}$ and orthogonal operators $O_{k}$. The optimal strategy satisfies the symmetry that for each such operator the set of operators $A_{k}$ contains as well the operator

$$
\tilde{A}_{k}=\sqrt{a_{k}} O_{k}+\left(\sqrt{b}_{k}-\sqrt{a_{k}}\right) O_{k} \bar{P}
$$

which employs the orthogonal complement $\bar{P}=\mathbb{1}_{\mathcal{H}}-P_{k}$. The optimal eavesdropping strategy associates a measurement outcome with each of the operators $A_{k}$ separately so that we do not need to employ any partitions $K^{(k)}$. For such an eavesdropping strategy the measured disturbance $D_{\text {fid }}$ is given by

$$
\begin{aligned}
D_{\text {fid }}=\sum_{i}\left[\frac{1}{4} \operatorname{Tr}_{\mathcal{H}}\left(\rho_{i} E_{i}\right)-\sum_{k \in K}\left(\frac{1}{4} \sqrt{a_{k} b_{k}} \operatorname{Tr}_{\mathcal{H}}\left(O_{k} \rho_{i} O_{k}^{\mathrm{T}} E_{i}\right)\right.\right. \\
\left.\left.+\frac{1}{4} \frac{\left(\sqrt{b_{k}}-\sqrt{a_{k}}\right)^{2}}{2}\left(\operatorname{Tr}_{\mathcal{H}}\left(O_{k} P_{k} \rho_{i} P_{k} O_{k}^{\mathrm{T}} E_{i}\right)+\operatorname{Tr}_{\mathcal{H}}\left(O_{k} \bar{P}_{k} \rho_{i} \bar{P}_{k} O_{k}^{\mathrm{T}} E_{i}\right)\right)\right)\right] .
\end{aligned}
$$

Here $E_{i}$ is the projection operator describing the effect of the polarisation analyser. For the one photon space we have $E_{i}=\rho_{i}$. The Shannon information is given by the expression

$$
\begin{aligned}
& I=\sum_{k} \frac{a_{k}+b_{k}}{2} {\left[1-\log \left(1+\eta_{k}^{2}\right)+\right.} \\
& \frac{1}{2\left(1+\eta_{k}^{2}\right)}\left\{\left(\eta_{k}^{2}+c_{k}-\eta_{k}^{2} c_{k}\right) \log \left(\eta_{k}^{2}+c_{k}-\eta_{k}^{2} c_{k}\right)\right. \\
&+\left(1-c_{k}+\eta_{k}^{2} c_{k}\right) \log \left(1-c_{k}+\eta_{k}^{2} c_{k}\right) \\
&+\left(\eta_{k}^{2}+d_{k}-\eta_{k}^{2} d_{k}\right) \log \left(\eta_{k}^{2}+d_{k}-\eta_{k}^{2} d_{k}\right) \\
&\left.\left.+\left(1-d_{k}+\eta_{k}^{2} d_{k}\right) \log \left(1-d_{k}+\eta_{k}^{2} d_{k}\right)\right\}\right]
\end{aligned}
$$

In this expression we used the definitions of the overlaps $c_{k}=\operatorname{Tr}_{\mathcal{H}}\left(\rho_{1} P_{k}\right)$ and $d_{k}=$ $\operatorname{Tr}_{\mathcal{H}}\left(\rho_{3} P_{k}\right)$ and of the characteristic parameters $\eta_{k}=\sqrt{\frac{a_{k}}{b_{k}}}$ with $\eta \in[0,1]$. For $\eta_{k}=1$ an operator $A_{k}$ takes the characteristics of the identity operator, which corresponds to non-interference of the eavesdropper, and for $\eta_{k}=0$ the eavesdropping strategy tends to a von Neumann projection measurement. The overlaps are restricted by the inequality

$$
\left(d_{k}-\frac{1}{2}\right)^{2}+\left(c_{k}-\frac{1}{2}\right)^{2} \leq \frac{1}{4}
$$

We can find the optimal choice of orthogonal operators $O_{k}$ and $P_{k}$. The optimal choices are given in a later section. As a result we find now for the disturbance the inequality

$$
D_{\text {fid }} \geq \sum_{k \in K} \frac{a_{k}+b_{k}}{2} \frac{1}{4} \frac{\left(1-\eta_{k}\right)^{2}}{1+\eta_{k}^{2}} .
$$


Note that the condition (1) implies that

$$
\sum_{k} \frac{a_{k}+b_{k}}{2}=1
$$

so that the expressions $\frac{a_{k}+b_{k}}{2}$ have the property of a probability. The Shannon information can be estimated by

$$
I \leq \sum_{k} \frac{a_{k}+b_{k}}{2} \frac{1}{2}\left(1-\log \left(1+\eta_{k}^{2}\right)+\frac{\eta_{k}^{2}}{1+\eta_{k}^{2}} \log \eta_{k}^{2}\right) .
$$

It can be shown that the optimal choice of the characteristic parameters $\eta_{k}$ is for them to take the same value $\tilde{\eta}$. The proof uses variation methods. Then we find the inequalities

$$
\begin{aligned}
D_{f i d} & \geq \frac{1}{4} \frac{(1-\tilde{\eta})^{2}}{1+\tilde{\eta}^{2}} \\
I & \leq \frac{1}{2}\left(1-\log \left(1+\tilde{\eta}^{2}\right)+\frac{\tilde{\eta}^{2}}{1+\tilde{\eta}^{2}} \log \tilde{\eta}^{2}\right) .
\end{aligned}
$$

If we actually measure the average error rate $D_{\text {fid }}$ and find the value $D_{m}$ we can bound the value of $\tilde{\eta}$ by

$$
\tilde{\eta} \geq \bar{\eta}:= \begin{cases}\frac{1-2 \sqrt{2} \sqrt{\left(1-2 D_{m}\right) D_{m}}}{1-4 D_{m}} & D_{m} \leq \frac{1}{4} \\ 0 & D_{m} \geq \frac{1}{4}\end{cases}
$$

which leads to the bound of the eavesdropper's Shannon information [7, 8] as

$$
I_{S} \leq \frac{1}{2}\left(1-\log \left(1+\bar{\eta}^{2}\right)+\frac{\bar{\eta}^{2}}{1+\bar{\eta}^{2}} \log \bar{\eta}^{2}\right) .
$$

It can be shown that this bound can be further estimated by the linear bound

$$
I \leq \frac{2}{\ln 2} D_{m}
$$

where $\ln 2$ is the natural logarithm of 2 . For small $D_{m}$ this bound is nearly as good as the bound (17) which will later be shown to be sharp. The sharp bound and the linear approximation are plotted in figure 3 as a function of the measured fidelity disturbance. Typical values for experimental realisations using the BB84 protocol achieve an error rate of $4 \%$ for $30 \mathrm{~km}$ or $1.5 \%$ for $10 \mathrm{~km}$ distance between sender and receiver.

\section{Privacy amplification}

For the purpose of secret communication the amount of Shannon information possibly leaked to an eavesdropper according to the previous estimate is far to high. However, 


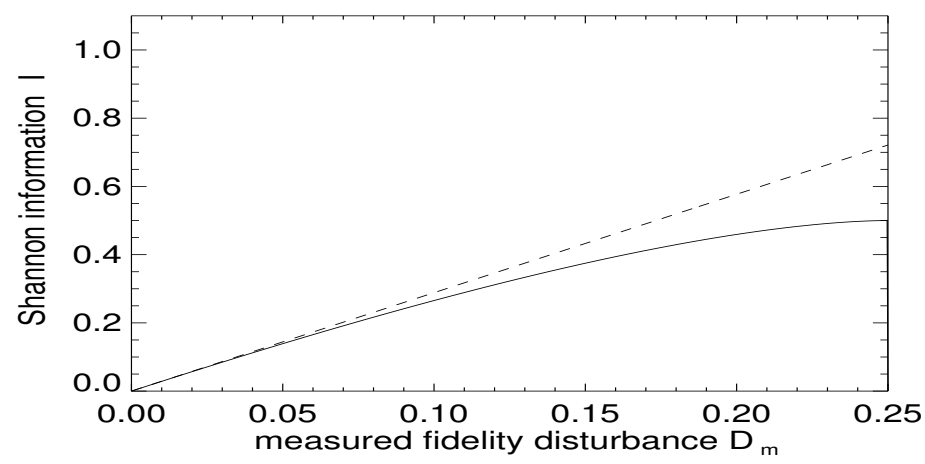

Figure 1: The bound on the Shannon information in the sharp (continuous line) and the linear bound (dashed line) as a function of the measured disturbance $D_{m}$.

making use of the technique of privacy amplification [9] it is possible to reduce Eve's total amount of Shannon information on the remaining key. For that the key has to be shortened using hash functions. The characteristic quantity for the fraction by which the key has to be shortened is the parameter $\tau_{1}$. If we shorten the key by the fraction $\tau_{1}$ then the eavesdropper is left at most with a Shannon information of 1 bit on the whole key. Each bit by which the key is is shortened additionally decreases this remaining Shannon information exponentially. The parameter $\tau_{1}$ can be expressed with the help of the collision probability $\left\langle p_{c}(y)\right\rangle_{y}$ as

$$
\tau_{1}=1+\log \left\langle p_{c}(y)\right\rangle_{y}^{\frac{1}{N}}
$$

The collision probability $\left\langle p_{c}(y)\right\rangle_{y}=\sum_{x} p(x \mid y)^{2}$ refers to the probability distribution $p(x \mid y)$ over all possible signal string $x$, conditioned on the event that the eavesdropper measured a particular string of measurement results $y$.

Before they can apply the technique of privacy amplification, the sender and the receiver have to perform some type of error correction on their shared key. We assume that this process can be done without the eavesdropper gaining any additional knowledge and without the creation of any correlations between the signals. A possible realisation would be to use block parity comparison where the compared parity bit is encoded using some short shared secret key from the same source which gives the key used in the authentication of the public channel. In this case the collision probability is given by

$$
\left\langle p_{c}(y)\right\rangle^{\frac{1}{n}}=\sum_{k, \alpha, \psi} \frac{p^{(c)}\left(\psi, k_{\alpha}\right)^{2}}{p^{(c)}\left(k_{\alpha}\right)},
$$

where the probabilities $p^{(c)}\left(\psi, k_{\alpha}\right)$ and $p^{(c)}\left(k_{\alpha}\right)$ now refer to the corrected key, and it takes only those signal transmissions which were correctly received into account. The 
joint probability distribution $p^{(c)}\left(\psi, k_{\alpha}\right)$ is given, with a normalisation constant $C$, by

$$
p^{(c)}\left(\psi, k_{\alpha}\right)=\frac{1}{C} \operatorname{Tr}_{\mathcal{H}}\left(A_{k} \rho_{\psi_{\alpha}} A_{k}^{\dagger} \rho_{\psi_{\alpha}}\right) .
$$

It is again possible to show general properties of the $A_{k}$ which lead to an optimal information gain by the eavesdropper, along with minimal disturbance of the signal transmission. The optimal $A_{k}$ can be shown to be real (in the real representation of the signal states) and to consist of symmetric or anti-symmetric matrices. The symmetric matrices can have eigenvalues of different or of the same sign so that they can be written as

$$
A_{k}^{( \pm)}=\sqrt{a_{k}} \mathbb{1}_{\mathcal{H}}-\left(\sqrt{a_{k}} \pm \sqrt{b_{k}}\right) P_{k}
$$

with the $a_{k}$ and $b_{k}$ satisfying, as before, $b_{k} \geq a_{k} \geq 0$ and $P_{k}$ is a projection operator. To each such operator the set of operators $A_{k}$ contains an operator

$$
\tilde{A}_{k}^{( \pm)}=\sqrt{a_{k}} \mathbb{1}_{\mathcal{H}}-\left(\sqrt{a_{k}} \pm \sqrt{b_{k}}\right) \overline{P_{k}}
$$

using the orthonormal complement $\overline{P_{k}}=\mathbb{1}_{\mathcal{H}}-P_{k}$. I refrain from giving to give the expressions for the collision probability and the disturbance $D_{\text {fid }}$ in the general form and give instead the forms optimised with respect to the choice of projection operators $P_{k}$. At this stage they are given with the help of the characteristic parameter $\eta_{k}$ which satisfies $\eta_{k}^{2}=\frac{a_{k}}{b_{k}}$ and takes values in the range $\eta_{k} \in[-1,1]$ which is in contrast to the calculations leading to the bound on the Shannon information. The disturbance satisfies the inequality

$$
D_{\text {fid }}=\sum_{k \in K^{( \pm)}} \frac{a_{k}+b_{k}}{2} \frac{1}{4} \frac{\left(\eta_{k}-1\right)^{2}}{\eta_{k}^{2}+1}+\sum_{k \in K^{(a)}} \frac{a_{k}+b_{k}}{2} \frac{1}{4} .
$$

where $K^{( \pm)}$is the index set of the symmetric operators and $K^{(a)}$ the index set of the anti-symmetric operators. The collision probability is bound by

$$
\left\langle p_{c}^{(c)}(y)\right\rangle^{\frac{1}{n}} \leq \frac{\sum_{k} \frac{a_{k}+b_{k}}{2} \frac{1}{2} \frac{1}{1+\eta_{k}^{2}} \frac{17+12 \eta_{k}+6 \eta_{k}^{2}+12 \eta_{k}^{3}+17 \eta_{k}^{4}}{3+2 \eta_{k}+3 \eta_{k}^{2}}}{\sum_{k} \frac{a_{k}+b_{k}}{2} \frac{3+2 \eta_{k}+3 \eta_{k}^{2}}{1+\eta_{k}^{2}}}
$$

We use again a variation method to show that the optimal eavesdropping strategy employs characteristic parameters $\eta_{k}$ with the same value $\tilde{\eta}$. Also it is clear that it is of disadvantage to the eavesdropper to use anti-symmetric operators $A_{k}$. This leads to the estimates

$$
D_{\text {fid }} \geq \frac{1}{4} \sum_{k \in K} \frac{(\tilde{\eta}-1)^{2}}{\tilde{\eta}^{2}+1}
$$


and

$$
\left\langle p_{c}^{(c)}(y)\right\rangle^{\frac{1}{N}} \leq \frac{1}{2} \frac{17+12 \tilde{\eta}+6 \tilde{\eta}^{2}+12 \tilde{\eta}^{3}+17 \tilde{\eta}^{4}}{\left(3+2 \tilde{\eta}+3 \tilde{\eta}^{2}\right)^{2}} .
$$

The measured disturbance $D_{m}$ leads to a bound on $\tilde{\eta}$ given by

$$
\tilde{\eta} \geq \bar{\eta}:=\left\{\begin{array}{ll}
\frac{1-2 \sqrt{2} \sqrt{\left(1-2 D_{m}\right) D_{m}}}{1-4 D_{m}} & D_{m} \leq \frac{1}{2} \\
-1 & D_{m} \geq \frac{1}{2}
\end{array} .\right.
$$

This finally allows us to bound the parameter $\tau_{1}(19)$ by the inequality 8

$$
\tau_{1} \leq \begin{cases}\log \left(\frac{17+12 \bar{\eta}+6 \bar{\eta}^{2}+12 \bar{\eta}^{3}+17 \bar{\eta}^{4}}{\left(3+2 \bar{\eta}+3 \bar{\eta}^{2}\right)^{2}}\right) & D_{m} \leq \frac{1}{3} \\ 1 & \frac{1}{3} \leq D_{m} \leq 1\end{cases}
$$

This bound is shown in figure 4 . Typical error rates in the BT experiment are $e \in$ $[0.01,0.05]$ which corresponds to $\tau_{1} \in[0.05,0.26]$.

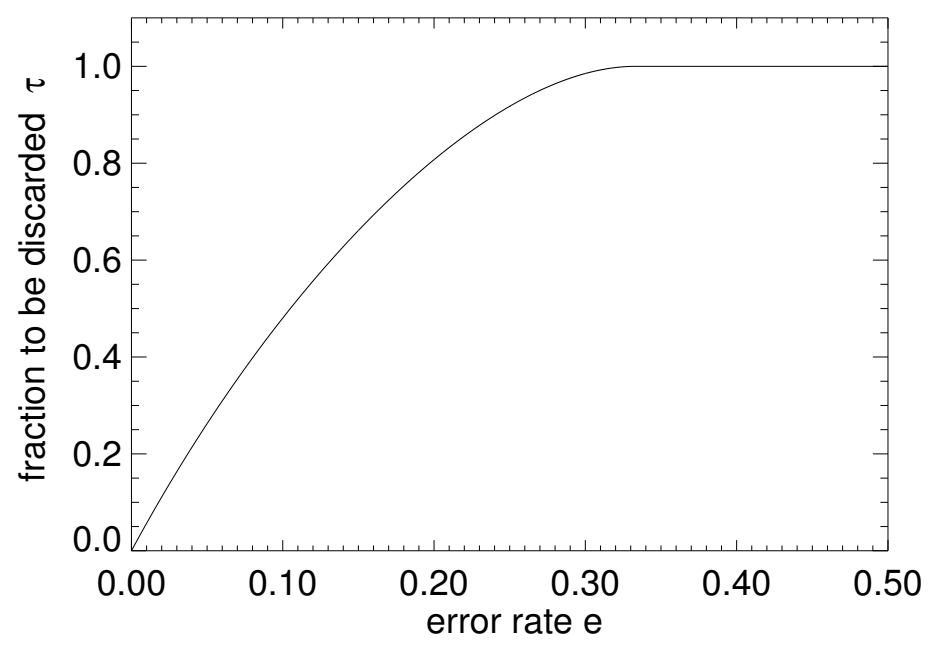

Figure 2: The parameter $\tau_{1}$ as a function of the error rate. The error rate $e$ is equal to the disturbance measure $D_{\text {fid }}$.

\section{$5 \quad$ Validity of the bounds}

The derivation of the bounds presented above assumes that the eavesdropper interacts with the signal photons but does not absorb them. In the experimental realisation, however, an absorption of about $90 \%$ is observed. The validity of the bounds can be extended 
to accommodate the possibility of absorption by re-defining the average error rate as refering only to those signals where the polarisation analyser successfully measured a signal. It can be shown that the eavesdropper cannot increase the trade-off between information and induced error rate by forwarding signal states to the receiver which contain more than one photon. The basic tool for this extension of the validity of the bounds is that one can show that each eavesdropper strategy is equivalent to an eavesdropper strategy which results in final states which are Fock states of fixed photon number.

\section{Delayed measurements}

The description of a delayed measurement needed here is that the eavesdropper has effectively two eavesdropping strategies at hand: one for each signal set of linear or circular polarisation. They are given by the A-operators $\left\{A_{k}\right\}_{k \in K}$ and $\left\{B_{l}\right\}_{l \in L}$ with two index sets $K$ and $L$ which are not necessarily of the same size. The strategies cannot be chosen independently of each other since they must be alternative descriptions of the quantum channel viewed as a non-selective measurement. This means that the equality

$$
\sum_{k \in K} A_{k} \rho A_{k}^{\dagger}=\sum_{l \in L} B_{l} \rho B_{l}^{\dagger}
$$

must hold for all density matrices $\rho$. An example of relations between the sets $\left\{A_{k}\right\}_{k \in K}$ and $\left\{B_{l}\right\}_{l \in L}$ satisfying this equality is the choice $B_{l}=\sum_{k} c_{l k} A_{k}$ with $\sum_{k} c_{l k} \bar{c}_{k n}=\delta_{l n}$. One can give a crude estimate of the Shannon information and of the collision probability because the disturbance is independent of the overlaps $c_{k}$ and $d_{k}$. To give the bounds let the eavesdropper choose the projection operators $P_{k}$ of the set of operators $A_{k}$ and $B_{k}$ independently. Quantum mechanics will put some restrictions on that relation so that the resulting bounds are no longer sharp. Thus the eavesdropper's Shannon information may increase by a factor 2 . The collision probability is bounded by

$$
\left\langle p_{c}^{(c)}(y)\right\rangle^{\frac{1}{n}} \leq \frac{1+\tilde{\eta}^{4}}{\left(1+\tilde{\eta}^{2}\right)^{2}}
$$

where $\tilde{\eta}$ is bounded by the measured disturbance $D_{\text {fid }}$ as given in (28). The resulting bound for the fraction $\tau_{1}$ of bits to be discarded during privacy amplification is plotted in figure 6. In this estimate we can prove security against eavesdropping as long as the error rate is less than $25 \%$. This result is likely to remain valid if we allow $n$-photon operations as in the previous chapter. Formal proof, however, should be postponed until a sharp bound for delayed choice eavesdropping strategies can be given. Clearer understanding of the restrictions imposed by (30) is essential for the derivation of the sharp bound.

\section{References}




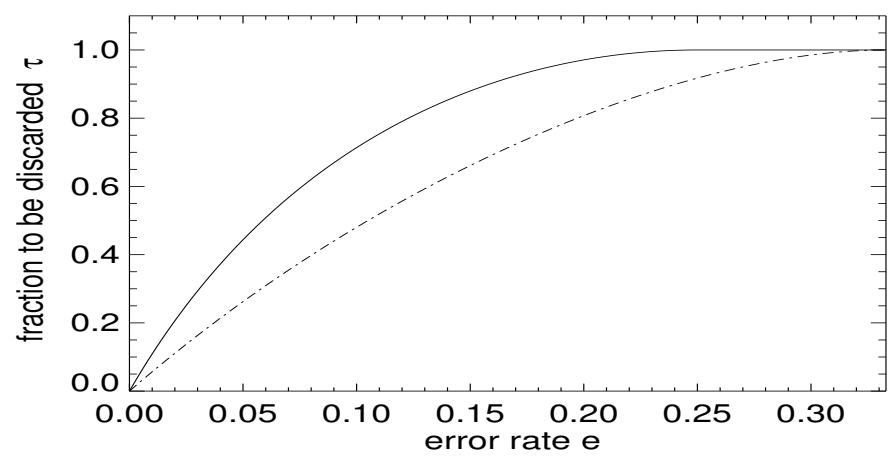

Figure 3: The crude bound of the fraction $\tau_{1}$ of bits to be discarded during privacy amplification allowing for delayed measurements (solid line). This is compared to the sharp bound for non-delayed measurements (dashed line).

[1] R. J. Hughes, D. M. Alde, P. Dyer, G. G. Luther, G. L. Morgan, and M. Schauer, "Quantum cryptography," Contemp. Phys. 36, 149-163 (1995).

[2] S. J. D. Phoenix and P. D. Townsend, "Quantum cryptography and secure optical communications," BT Technol. J. 11, 65-75 (1993).

[3] S. J. D. Phoenix and P. D. Townsend, "Quantum cryptography - how to beat the code breakers using quantum-mechanics," Contemp. Phys. 36, 165-195 (1995).

[4] C. H. Bennett and G. Brassard, "Quantum cryptography: Public key distribution and coin tossing.," In Proceedings of IEEE International Conference on Computers, Systems, and Signal Processing, Bangalore, India, (IEEE, New York, 1984) pp. 175179 .

[5] B. Huttner and A. K. Ekert, "Information gain in quantum eavesdropping," J. Mod. Opt. 41, 2455-2466 (1994).

[6] A. K. Ekert and B. Huttner and G. M. N. Palma and A. Peres, "Eavesdropping on quantum-cryptographical systems," Phys. Rev. A 50, 1047-1056 (1994).

[7] N. Lütkenhaus, "Security against eavesdropping in quantum cryptography," Phys. Rev. A 54, 97 (1996).

[8] N. Lütkenhaus, Ph.D. thesis, University of Strathclyde, 1996.

[9] C. H. Bennett, G. Brassard, C. Crépeau, and U. M. Maurer, "Generalized privacy amplification," IEEE Trans. Inf. Theo. 41, 1915 (1995). 